\title{
ABORDAGEM MATEMÁTICA E NUMÉRICA DO ESCOAMENTO BIFÁSICO EM MEIOS POROSOS
}

\author{
A. T. S. SOUZA ${ }^{1 *}$, J. T. CEVOLANI ${ }^{1}$, A. P. MENEGUELO ${ }^{1}$ e D. da C. RIBEIRO ${ }^{1}$ \\ ${ }^{1}$ Universidade Federal do Espírito Santo, Departamento de Engenharias e Tecnologia \\ *E-mail para contato: aletssouza@gmail.com
}

\begin{abstract}
RESUMO - A simulação numérica do escoamento bifásico em meios porosos é importante na engenharia de petróleo por auxiliar na previsão dos volumes de óleo a serem recuperados. Atualmente grande parte dos códigos computacionais disponíveis para análise do escoamento em meios porosos é comercial e não disponibiliza os códigos para implementações adicionais. Desta forma, propor um modelo matemático e desenvolver sua modelagem numérica possibilita a implementação do código e que análises especificas sejam realizadas. Por essa razão, este artigo objetiva desenvolver a modelagem numérica para um escoamento bifásico, imiscível, moderadamente compressível e unidimensional entre água e óleo, desconsiderando as contribuições das forças gravitacionais. A abordagem IMPES foi adotada como estratégia de resolução devido sua simplicidade e difusão no meio. Com as equações desenvolvidas foi proposto um algoritmo que permite avaliar as variações na pressão e na saturação dos fluidos envolvidos no decorrer de um processo de injeção de água.
\end{abstract}

\section{INTRODUÇÃO}

Um dos objetivos da simulação numérica na engenharia de reservatórios é prever o comportamento de fluidos durante um determinado processo, podendo ser empregada para avaliar a eficiência de métodos de recuperação (Heinemann, 2005). Apesar de apresentar soluções aproximadas, com os avanços tecnológicos, são elaborados modelos cada vez mais detalhados e sofisticados que capturam com maior precisão a física do escoamento dos fluidos (Gerritsen e Durlofsky, 2005).

As equações adotadas tiveram como base o modelo black oil (Chen et al, 2006) e buscaram representar um processo de injeção de água para recuperação de óleo armazenado em rochas reservatório desconsiderando a ação da força. Para resolução do sistema de equações gerado optou-se por implementar a estratégia IMPES (Implicit Pressure Explicit Saturation).

Na metodologia IMPES, são resolvidas duas equações, a primeira de forma implícita, para o cálculo da pressão de um dos fluidos, e a segunda de forma explícita, para o cálculo da saturação de um dos fluidos. As pressões e saturações dos demais fluidos são obtidas pela adição dos conceitos de saturação total e pressão capilar (Franc et al, 2016).

Dentre os autores que estudaram o método IMPES, Kou e Sun (2004) propuseram avaliar a pressão capilar no passo de tempo atual ao invés do passo de tempo anterior como é 
feito na forma clássica. Ao comparar as duas abordagens, para um mesmo passo de tempo, em um escoamento bifásico e imiscível, a nova versão apresentou melhor convergência diminuindo o custo computacional.

Chen et al (2004) observaram que as mudanças na pressão ocorrem mais devagar do que na saturação e sugeriram adotar passos de tempo diferentes para cada equação, sendo o da pressão maior do que da saturação. Comparando o novo IMPES com o método SS (Sequential Solution) (Chen et al 2003), constatou-se a eficiência e estabilidade da abordagem, mesmo em problemas com elementos de tamanho pequeno.

Neste artigo será proposto um algoritmo para a resolução de um escoamento bifásico imiscível, considerando a presença das forças capilares, com o objetivo de avaliar as variações na pressão e na saturação dos fluidos envolvidos no decorrer de um processo de injeção de água.

\section{ABORDAGEM MATEMÁTICA}

As Equações 1 e 2 foram obtidas da junção das equações de conservação de massa, lei de Darcy e equações de estado, desconsiderando os efeitos gravitacionais (Kleppe, 2016) e descrevem um escoamento bifásico (água e óleo), imiscível, moderadamente compressível e unidimensional:

$$
\begin{aligned}
& \frac{\partial}{\partial x}\left(\frac{k k_{r w}}{\mu_{w} B_{w}} \frac{\partial P_{w}}{\partial x}\right)-q_{w}=\frac{\partial}{\partial t}\left(\frac{\phi S_{w}}{B_{w}}\right) \\
& \frac{\partial}{\partial x}\left(\frac{k k_{r o}}{\mu_{o} B_{o}} \frac{\partial P_{o}}{\partial x}\right)-q_{o}=\frac{\partial}{\partial t}\left(\frac{\phi S_{o}}{B_{o}}\right)
\end{aligned}
$$

Onde: $o$ corresponde a fase óleo, $w$ corresponde a fase água, $k$ é a permeabilidade absoluta, $k_{r o}$ é a permeabilidade relativa do óleo, $k_{r w}$ é a permeabilidade relativa da água, $\mu_{o}$ é a viscosidade do óleo, $\mu_{w}$ é a viscosidade da água, $P_{o}$ é a pressão do óleo, $P_{w}$ é a pressão da água, $q_{o}$ é a vazão de injeção/produção de óleo, $q_{w}$ é a vazão de injeção/produção de água, $\phi$ é a porosidade do meio, $S_{o}$ é a saturação do óleo e $S_{w}$ é a saturação da água.

Nesse sistema tem-se quatro incógnitas: $P_{o}, P_{w}, S_{o}$ e $S_{w}$ e apenas duas equações. Para resolvê-lo adicionou-se os conceitos de saturação total $\left(S_{t}\right)$ (Equação 3$)$ e pressão capilar $\left(P_{c}\right)$ (Equação 4) (Kleppe, 2016):

$$
\begin{aligned}
& S_{t}=S_{o}+S_{w}=1 \\
& P_{c}=P_{o}-P_{w}
\end{aligned}
$$

\section{ABORDAGEM NUMÉRICA}

A discretização das Equações 1 e 2 teve como base o método de diferenças finitas, que utiliza expansão em séries de Taylor para transformar as equações integráveis em diferenças discretas (Kamyabi, 2014). Considerando porosidade constante e o fator volume-formação da água constante, foram obtidas as Equações 5 (água) e 6 (óleo): 


$$
\begin{gathered}
T_{w_{i+\frac{1}{2}}}\left(P_{w_{i+1}}-P_{w_{i}}\right)+T_{w_{i-\frac{1}{2}}}\left(P_{w_{i-1}}-P_{w_{i}}\right)+q_{w_{i}}=\frac{V_{i} \phi}{B_{w}} \frac{\left(S_{w_{i}}^{t+1}-S_{w_{i}}{ }^{t}\right)}{\Delta t} \\
T_{o_{i+\frac{1}{2}}}\left(P_{o_{i+1}}-P_{o_{i}}\right)+T_{o_{-\frac{1}{2}}}\left(P_{o_{i-1}}-P_{o_{i}}\right)+q_{o_{i}} \\
=\frac{V_{i} \phi}{B_{o_{i}}} \frac{\left(S_{o_{i}}^{t+1}-S_{o_{i}}^{t}\right)}{\Delta t}+V_{i} \phi S_{o_{i}}^{t} \frac{\partial\left(1 / B_{o_{i}}\right)}{\partial P_{o_{i}}} \frac{\left(P_{o_{i}}^{t+1}-P_{o_{i}}^{t}\right)}{\Delta t}
\end{gathered}
$$

Onde: $V_{i}$ é o volume e T é chamado de transmissibilidade, definido como:

$$
T=\frac{k k_{r} A}{\mu B \Delta x}
$$

Onde: $A$ é a área da seção perpendicular ao escoamento

\subsection{METODOLOGIA IMPES}

Aplicando o conceito de $P_{c}$ na Equação (6) e desconsiderando o gradiente da pressão capilar (Rosa et al, 2006) tem-se a equação discretizada para o óleo (Equação 8),

$$
\begin{aligned}
T_{o_{i+\frac{1}{2}}}\left(P_{w_{i+1}}-\right. & \left.P_{w_{i}}\right)+T_{o_{i-\frac{1}{2}}}\left(P_{w_{i-1}}-P_{w_{i}}\right)+q_{o_{i}} \\
& =\frac{V_{i} \phi}{B_{o_{i}}} \frac{\left(S_{o_{i}}^{t+1}-S_{o_{i}}^{t}\right)}{\Delta t} \\
& +V_{i} \phi S_{o_{i}} \frac{\partial\left(1 / B_{o_{i}}\right)}{\partial P_{o_{i}}}\left(\frac{\partial P_{c_{i}}}{\partial S_{o_{i}}^{t}} \frac{\left(S_{o_{i}}^{t+1}-S_{o_{i}}^{t}\right)}{\Delta t}+\frac{\left(P_{w_{i}}^{t+1}-P_{w_{i}}^{t}\right)}{\Delta t}\right)
\end{aligned}
$$

Somando as Equações 5 e 8, mantendo apenas as pressões da água como incógnitas,

$$
\begin{aligned}
& B_{w} \Gamma_{2}\left[T_{w_{i+\frac{1}{2}}}^{t}\left(P_{w_{i+1}}^{t+1}-P_{w_{i}}^{t+1}\right)+T_{w_{i-\frac{1}{2}}}^{t}\left(P_{w_{i-1}}^{t+1}-P_{w_{i}}^{t+1}\right)+q_{w_{i}}^{t}\right]+T_{o_{i+\frac{1}{2}}}^{t}\left(P_{w_{i+1}}^{t+1}-P_{w_{i}}^{t+1}\right) \\
& +T_{o-\frac{1}{2}}^{t}\left(P_{w_{i-1}}^{t+1}-P_{w_{i}}^{t+1}\right)+q_{o}^{t}=\Gamma_{1} \frac{\left(P_{w_{i}}^{t+1}-P_{w_{i}}^{t}\right)}{\Delta t} \\
& \Gamma_{1}=V_{i} \phi S_{o_{i}}{ }^{t} \frac{\partial\left(1 / B_{o_{i}}\right)}{\partial P_{o_{i}}} \\
& \Gamma_{2}=\frac{1}{B_{o_{i}}}+\Gamma_{1} \frac{\partial P_{c_{i}}}{\partial S_{o_{i}}^{t}}
\end{aligned}
$$

Reorganizando a Equação 9,

$$
\begin{aligned}
& a_{i}^{t+1} P_{w_{i-1}}^{t+1}+b_{i}^{t+1} P_{w_{i}}^{t+1}+c_{i}^{t+1} P_{w_{i+1}}^{t+1}=d_{i}^{t+1} \\
& a=T_{w_{i-\frac{1}{2}}}^{t}+T_{o_{i-\frac{1}{2}}}^{t} \\
& b=-B_{w} \Gamma_{2}\left(T_{w_{i+\frac{1}{2}}}^{t}+T_{w_{i-\frac{1}{2}}}^{t}\right)-\left(T_{o_{i+\frac{1}{2}}}^{t}+T_{o_{i-\frac{1}{2}}}^{t}\right)-\frac{\Gamma_{1}}{\Delta t}
\end{aligned}
$$


$c=T_{w_{i+\frac{1}{2}}^{t}}^{t}+T_{o_{i+\frac{1}{2}}^{t}}^{t}$

$d=-B_{w} \Gamma_{2} q_{w i}^{t}-q_{o}^{t}-\Gamma_{1} \frac{P_{w_{i}}^{t}}{\Delta t}$

A Equação (12) é aplicada em todos os elementos gerando um sistema linear tridiagonal, por meio do qual, serão obtidas as pressões da água em cada elemento. Este sistema pode ser resolvido por métodos diretos (Eliminação de Gauss, Decomposição LU, Eliminação de Gauss-Jordan, etc.) ou interativos (Método de Newton, Método da bisseção, etc.). Neste trabalho será utilizado o método direto de Thomas (Gilat e Subramaniam, 2008). A saturação da água (Equação 17) é calculada rearranjando a equação discretizada da água (Equação 5):

$$
S_{w_{i}}^{t+1}=S_{w_{i}}^{t}+\frac{B_{w} \Delta t^{t+1}}{V_{i} \phi}\left[T_{w_{i+\frac{1}{2}}{ }^{t}}\left(P_{w_{i+1}}^{t+1}-P_{w_{i}}^{t+1}\right)+T_{w_{i-\frac{1}{2}}}^{t}\left(P_{w_{i-1}}^{t+1}-P_{w_{i}}^{t+1}\right)+q_{w_{i}}^{t}\right]
$$

As pressões do óleo podem ser determinadas pela Equação 4 e as saturações do óleo são calculadas com a Equação 3.

\section{RESULTADOS}

A partir das equações desenvolvidas, foi proposto o seguinte algoritmo mostrado na Figura 1.

Figura 1 - Escopo da função IMPES.

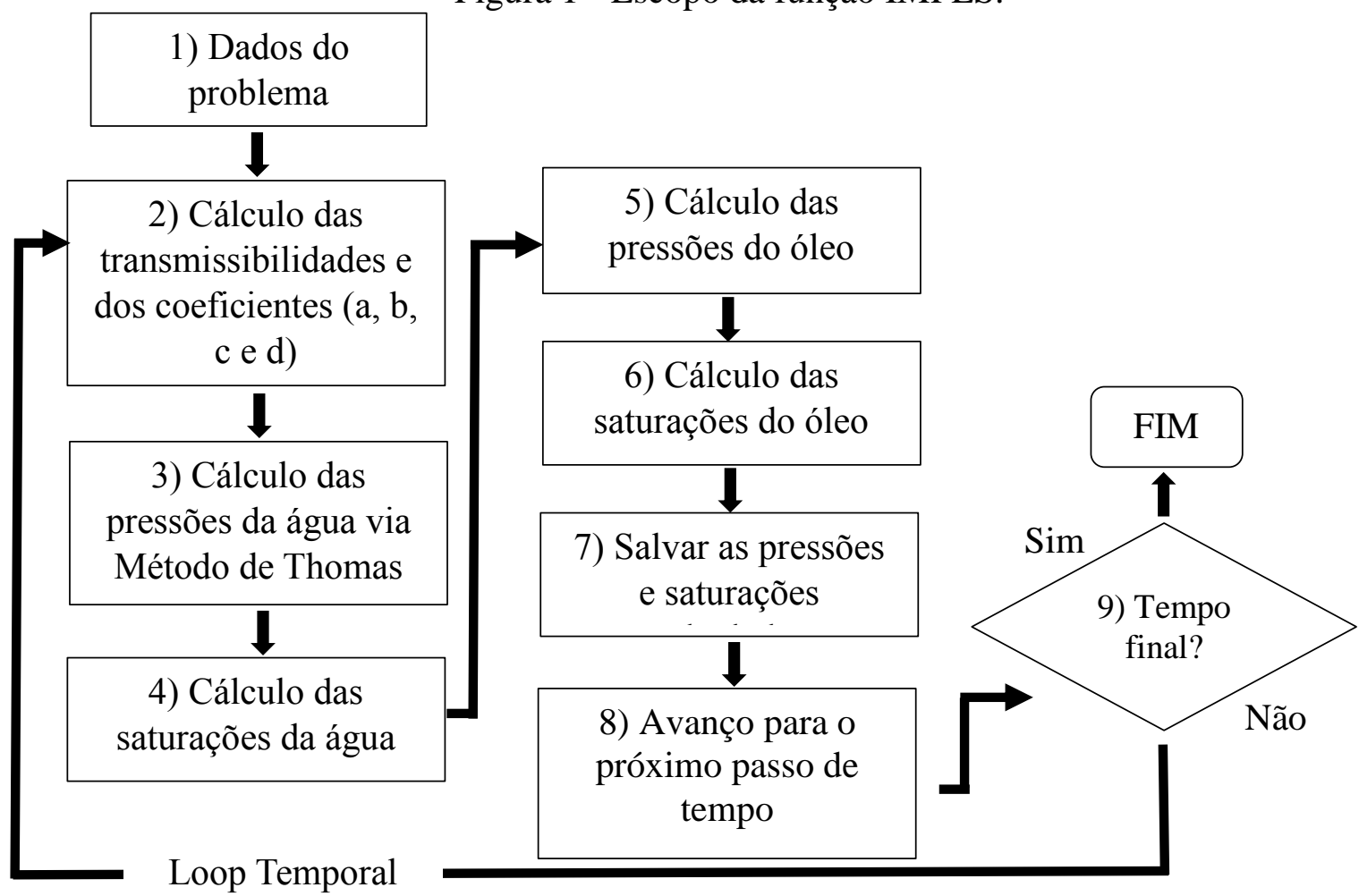


O item 1) recebe as informações iniciais do problema como: dados dos fluidos (viscosidade e fator volume-formação), dados do reservatório (porosidade, permeabilidade absoluta e dimensões), dados gerais da simulação (pressão inicial, pressão capilar inicial, saturação inicial de água, vazões de injeção e produção, número de elementos e tempo total),

Em 2), realiza-se o cálculo das transmissibilidades (Equação 7) e das variáveis a, b, c e d (Equações 13-16, respectivamente). Na etapa 3), o método de Thomas não requer a construção de matrizes, portanto as variáveis podem ser utilizadas na forma de vetores, o que reduz o tempo de simulação, mesmos assim, essa fase é a que demanda mais tempo por envolver resolver a Equação 12 de forma implícita. Nas demais etapas, os cálculos são rápidos e resolvidos de forma explícita. De forma geral, o custo computacional total será influenciado pelo grau de refinamento da malha e pelo tempo total de simulação.

\section{CONSIDERAÇÕES FINAIS}

O modelo desenvolvido deve ser aplicado respeitando-se as simplificações adotadas e de posse das informações necessárias (dados dos fluidos, dados do reservatórios e dados gerais). O método direto de Thomas pode ser substituído por outro método direto ou por métodos interativos. Lembrando que para métodos interativos deve atentar para critérios de convergência.

Esse algoritmo pode ser empregado em qualquer linguagem computacional. E, possui baixo custo computacional, em comparação com o uso de métodos interativos no cálculo implícito da pressão.

\section{REFERENCIAS}

CHEN, Z.; HUAN, G. e MA, Y. Computational methods for multiphase flows in porous media. Society for Industrial and Applied Mathematics. ISBN 0-89871-606-3. 2006.

CHEN, Z.; LI, B. e. HUAN, G. An Improved IMPES Method for Two-Phase Flow in Porous Media. Kluwer Academic Publishers. Holanda. 2004.

CHEN, Z.; LI, B. e. HUAN, G. The sequential method for the black-oil reservoir simulation on unstructured grids. Journal of Computational Physics. Vol. 192. 36-72. 2003.

FRANC, J.; HORGUE, P.; GUIBERT, R. e DEBENEST, G. Benchmark of different CFL conditions for IMPES. 2016. Disponível em: http://www.sciencedirect.com/science/article/pii/S1631072116300821. Acesso em: 22 de setembro de 2016.

GERRITSEN, M. G. e DURLOFSKY, L. J. Modeling Fluid Flow in oil Reservoirs. 2005. Disponível

em: http://www.annualreviews.org/doi/abs/10.1146/annurev.fluid.37.061903.175748?journa lCode=fluid. Acesso em: 22 de setembro de 2016. 
GILAT, A. e SUBRAMANIAM, V. Métodos numéricos para engenheiros e cientistas: uma introdução com aplicações usando o MATLAB. Ed: Bookman. Porto Alegre, Brasil. ISBN 978-85-7780-297-5. 2008.

HEINEMANN, Z. E. Introduction to Reservoir Simulation. 2005. Disponível em: https://pure.unileoben.ac.at/portal/files/561651/Introduction_to_reservoir_simulation.pd f. Acesso em: 22 de setembro de 2016.

KAMYABI, F. Multiphase Flow in Porous Media. Dissertação (Mestrado em Engenharia de Petróleo). Departamento de Engenharia de Petróleo e Geofísica Aplicada. Norwegian University of Science and Technology. Trondheim, Noruega. 2014.

KLEPPE, J. Saturated oil-water simulation, IMPES solution. 2016. Disponível em: http://www.ipt.ntnu.no/ kleppe/TPG4160/note6.pdf. Acesso em: 22 de setembro de 2016.

KOU, J e SUN, S. On Iterative IMPES Formulation for Two-phase Flow with Capillarity in Heterogeneous Porous Media. International Journal of Numerical Analysis and Modeling. Vol. 1. 20-40. 2004.

MARCONDES, F.; MALISKA, C. R. E ZAMBALDI, M. C. A Comparative Study of Implicit and Explicit Methods Using Unstructured Voronoi Meshes in Petroleum Reservoir Simulation. Journal of the Brazilian Society of Mechanical Science and Engineer. Vol. 31. 353-361. 2009.

MATHEWS, J. H. e FINK, K.D. Numerical Methods using MATLAB. $3^{\circ}$ edição. Prentice Hall. Upper Saddle River. NJ 07458. 1999.

ROSA, A. J.; CARVALHO, R. S.; XAVIER, J. A. D. Engenharia de Reservatórios de Petróleo. Ed: Interciência. Rio de Janeiro. 2006. 